\title{
Cambios importantes en la cobertura de la tierra y análisis de transiciones sistemáticas en un sector del occidente de Honduras
}

\author{
María Cristina Pineda de Carías, German Flores, \\ Mirza Castro, Pedro Jiménez, Gerardo Reyes \\ y Enrique López
}

\section{Resumen}

El objetivo fue aplicar tecnologías de información geográfica para identificar cambios importantes en cobertura de la tierra y, analizar señales dominantes ligadas a procesos de cambios sistemáticos en el occidente de Honduras. Se analizaron períodos antes y después del huracán Mitch que afectó Centroamérica en 1998. Se utilizaron mapas de 1991, 1995, 2003 y 2006 clasificados en: bosque mixto, pino, matorral, pastos, agricultura, urbano y suelo desnudo. Los de 1991 y 1995 y de 2003 y 2005 se compararon para construir matrices de tabulación cruzada que se analizaron separadamente para identificar persistencia, ganancias y pérdidas por categorías. Se calculó cambio neto, intercambio, cambio total; y las transiciones entre categorías. Como resultado se encontró que entre 1991-1995 el mayor cambio neto fue en agricultura (9.54\%), matorral (6.07\%) y pino (4.92\%). Para 2003-2006, en matorral (5.54\%), pasto (4.39\%) y bosque mixto (3.13\%). Entre 1991-1995 el intercambio se dio en la mayoría de las categorías: bosque mixto $(20.15 \%)$, agricultura (18.86\%), matorral $(13.56 \%)$, pino $(12.32 \%)$ y suelo desnudo $(11.96 \%)$; y entre 2003-2006 en: agricultura (23.98\%), pino (15.30\%), suelo desnudo (14.77\%), matorral (13.47) y bosque mixto (8.22\%). Los resultados evidencian una alta dinámica de cambio del paisaje, 52.57\% entre 1991-1995 y 48.78\% entre 2003-2006. Las principales transiciones sistemáticas entre 1991-1995 fueron: de bosque mixto a pino y de matorral a agricultura, las primeras debidas a la expulsión de grandes aserraderos de la zona; las segundas al avance de la frontera agrícola. Entre 20032006 las transiciones se dieron: de pino a bosque mixto y de matorral a pino, las primeras debidas a la importancia económica que adquirieron otras especies, las segundas al agotamiento de capacidad productiva de matorrales que pasaron a especies de mayor valor económico, como el pino. Estas últimas tendencias revelan 
potencialidad de regeneración natural del bosque e intencionalidades humanas de protección y económicas.

Palabras clave: Cobertura de la tierra; cambios en categorías; transiciones sistemáticas; Honduras: Occidente.

\section{Abstract}

The aim was to apply geographic information technologies to identify significant changes in land cover and analyze dominant signals linked to systematic processes of changes in western Honduras. Periods were analyzed before and after Hurricane Mitch that hit Central America in 1998. We used maps of 1991, 1995, 2003 and 2006 classified in: mixed forest, pine, shrubs, herbaceous, agriculture, urban and bare soil. The 1991 and 1995 and 2003 and 2005 were compared to build cross-tabulation matrices that were analyzed separately to identify persistence, gains and losses by category. We calculated net change, swap, total change; and the transitions between categories. As a result it was found that for 1991-1995 the largest net change was in agriculture (9.54\%), shrubs (6.07\%) and pine (4.92\%); for 20032006 , in shrubs (5.54\%), herbaceous (4.39\%) and mixed forest $(3.13 \%)$. Between 1991 -1995 the swap occurred in most of the categories: mixed forest $(20.15 \%)$, agriculture (18.86\%), shrubs (13.56\%), pine (12.32\%) and bare soil $(11.96 \%)$; and between $2003-2006$ in: agriculture (23.98\%), pine (15.30\%), bare soil (14.77\%), scrubs (13.47) and mixed forest (8.22\%). The results show a high dynamic landscape change, $52.57 \%$ in $1991-1995$ and $48.78 \%$ in $2003-2006$. The main systematic transitions between 1991 -1995 were: mixed forest to pine and shrubs to agriculture, the first due to the expulsion of large sawmills in the area, the latter to the advance of the agricultural frontier. Between $2003-2006$ the main systematic transitions were: pine to mixed forest and shrubs to pine, the first due to the economic importance that there acquired other species, the second to the depletion of productive capacity of shrubs that became most economically valuable species, as pine. These latest trends reveal potential of natural regeneration of the forest and human protection and economic intentions. 
Keywords: Land cover; categorical changes; systematic transitions; Honduras: West.

María Cristina Pineda de Carías (mcpinedacarias@gmail.com), Universidad Nacional Autónoma de Honduras, Facultad de Ciencias Espaciales. German Flores (german.flores@. fao.org.hn); Mirza Castro (mirza.castro@fao.org); Pedro Jiménez (pedro.jimenezPJ@. gmail.com); Gerardo Reyes (gareyesn@yahoo.com); Enrique López (enriqueauxume17@ yahoo.es). Programa Especial para la Seguridad Alimentaria (PESA/FAO). 


\section{INTRODUCCION}

En 1998 Mitch, primero como un huracán y luego como una tormenta tropical azotó severamente el territorio centroamericano particularmente a Honduras. Para detectar los cambios en la cobertura de la tierra antes y después de Mitch sobre un sector del occidente de Honduras, se realizó un estudio abarcando los años de 1991 a 2006 (Pineda de Carías, Ochoa, \& Corrales, 2012). Se utilizaron cuatro imágenes LandSat del sector (resolución espacial de 28.5 metros) de diferentes fechas comprendiendo un período de siete y medio años antes y después Mitch. La metodología empleada incluyó, desde la selección de las imágenes de fechas y características apropiadas, el pre procesamiento para realizar correcciones geométricas y radiométricas, la delimitación del área de estudio y acopio de información relevante de la zona, la obtención de una leyenda basada en el Sistema de Clasificación de Cobertura de la Tierra de la FAO/UNEP, la realización de clasificación supervisada de las cuatro imágenes; la verificación de resultados mediante el uso de radiometría de campo y técnicas estadísticas, terminando con el análisis multitemporal para la detección de cambios. Como resultado importante se detectó que antes de Mitch, el tamaño del bosque se mantuvo en un $30 \%$, pero que después había disminuido de $25 \%$ a $20 \%$. Se atribuyó al marcado avance de la frontera agrícola a expensas de la disminución de la cobertura de bosques del sector; la agricultura se extendía más allá de las laderas, internándose en la zona de bosques. Por otro lado, se presentó como resultado que la arboleda de pinos que mostraba una tendencia decreciente antes de Mitch, de $20 \%$ a $15 \%$, había revertido esta tendencia de $20 \%$ a $22 \%$, con evidentes signos de recuperación; que también los matorrales, que antes de Mitch mostraron disminución de $18 \%$ a $12 \%$, mostraron una recuperación de 11 a 18\%; y que la agricultura, antes y después de Mitch mostró una tendencia creciente, pasando antes de $20 \%$ a $30 \%$, y después de $25 \%$ a $27 \%$. Como una de las conclusiones se presentó que la agricultura practicada en el sector estudiado era mixta, no solamente en cuanto al tipo de cultivos, sino por las prácticas culturales. Una parte de las zonas agrícolas se presentaban como zonas sin cambio, mas permanentes, otras sin embargo, las que usaban prácticas más tradicionales mostraban decrementos, al cambiar continuamente el tipo y los lugares de cultivo.

Los resultados de este estudio llamaron mucho la atención de la comunidad de investigadores y técnicos de la zona, que pronto demandaron profundizar más en los resultados. Fue así que buscando una aproximación diferente, encontramos la metodología propuesta por Pontius et al (Pontius, Shusas, \& McEachem, 
2004) para detectar cambios importantes en las categorías de la cobertura de la tierra a partir de la persistencia. Con métodos estadísticos nuevos como ellos dicen, se pueden identificar señales de procesos sistemáticos dentro de un patrón de cambio de la tierra, ayudando a buscar las señales más fuertes de cambios de la cobertura de la tierra sistemáticos y ultimadamente ligar los patrones a los procesos. En términos prácticos el análisis del cambio de la cobertura de la tierra se hace, obteniendo primero un mapa de la fecha 1 y de la fecha 2; examinando los cambios con una matriz de transición para identificar las transiciones más importantes; y luego investigar los procesos que generaron las transiciones.

Este trabajo ha tenido como objetivo aplicar tecnologías de información geográfica para identificar cambios importantes en la cobertura de la tierra y, analizar señales dominantes ligadas a procesos de cambios sistemáticos en ocho municipios de los departamentos de Copan y Lempira, del occidente de Honduras, analizando dos período de tiempo, uno antes y otro después del huracán Mitch. Se utilizó el Método de Pontius et al para realizar diferentes cálculos de persistencia y cambio total de las categorías de clasificación, tratando de responder a las siguientes preguntas: ¿cuál es el cambio neto de cada categoría, cuáles son las ganancias, pérdidas y el intercambio de cada categoría, cuáles son las mayores transiciones sistemáticas entre categorías?

Como resultado se encontró evidencia de una alta dinámica de cambio del paisaje, $52.57 \%$ entre $1991-1995$ y $48.78 \%$ entre $2003-2006$. Que las principales transiciones sistemáticas entre 1991-1995 fueron: de bosque mixto a pino y de matorral a agricultura. Entre 2003-2006 las principales transiciones se dieron: de pino a bosque mixto y de matorral a pino. Estas últimas tendencias revelan potencialidad de regeneración natural del bosque e intencionalidades humanas de protección y económicas.

\section{METODOLOGIA}

\section{Área de estudio y base de datos}

El área de estudio es un sector cuadrado de 23 kilómetros de lado, de 53,160 hectáreas que abarca el lado sureste del departamento de Copán, específicamente en las colindancias de los municipios de Santa Rosa de Copán, San Juan de Opoa y Cucuyagua, y la parte noreste del departamento de Lempira en sus municipios de Lepaera, Talgua, Las Flores y Gracias. El cuadrado está delimitado 
por las coordenadas: $301,084 \mathrm{~m}$ E y $1627,720 \mathrm{~m} \mathrm{~N}$ (esquina superior izquierda); y $324,111 \mathrm{~m}$ E y $1614,692 \mathrm{~m} N$ (esquina inferior derecha). Dentro de la imagen LandSat P019R050, el sector se escogió por ser representativo del paisaje del occidente de Honduras: con altas montañas, coberturas boscosas, ríos, áreas urbanas y agrícolas (Pineda Portillo, 2008).

Se utilizó cartografía temática de cuatro mapas de 1991, 1995, 2003 y 2006 clasificadas todos con los mismos criterios en siete clases: bosque mixto, pino, matorral, pastos, agricultura, urbano y suelo desnudo (Pineda de Carías, Ochoa, \& Corrales, 2012). En la Figura 1 se presenta un diagrama temporal, mostrando que el período estudiado abarcó quince años, distribuidos siete y medio años antes y después de Mitch.

FIGURA 1. Localización temporal de los mapas clasificados utilizados.

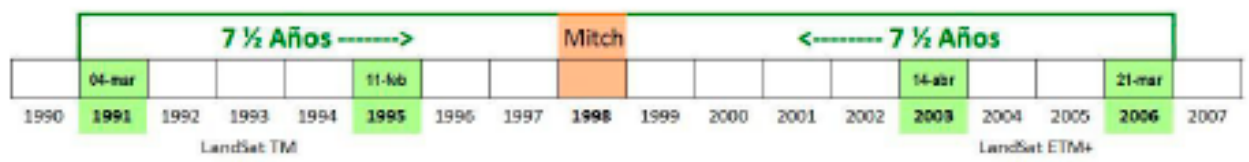

La leyenda de clasificación de los mapas temáticos utilizados incluyó siete clases:

1) Bosque mixto. Clase compuesta de dos tipos de cobertura: a) árboles, de cobertura cerrada, altura $>30-14 \mathrm{~m}$, distribución espacial: fragmentada; hoja ancha; ejemplo: roble, encino, laurel; b) árboles, de cobertura dispersa de 15 a 4\%; altura >30 - 14m; hoja aciculada; ejemplo: pinos.

2) Bosque de pino. Clase de un solo tipo de cobertura. Bosque, de cobertura abierta de 65 a 15\%; >30-14m; hoja acicular; ejemplo: pino.

3) Matorral. Clase de un solo tipo de cobertura. Arbustos, de cobertura abierta de 65 a $15 \%$; altura de 5 a $0.5 \mathrm{~m}$; hoja ancha.

4) Pastos. Clase de dos tipos de cobertura herbácea, en un campo de grande a mediano, distribución espacial continua; con un cultivo adicional, herbáceo terrestre, simultáneo; regado con agua de lluvia; a) uno, cultivo de rotación cambiante, parte de un proceso agrícola; b) el otro, de cultivo permanente. 
5) Agricultura. Clase de por lo menos cinco tipos de cobertura: a) arbustos, en huertos u otro tipo de plantación, en campo de grande a mediano, distribución espacial continúa, con dos cultivos adicionales de arbustos simultáneos, regados con lluvia, cultivo permanente, por ejemplo, café; b) arbustos, plantación; en campo de grande a mediano, distribución espacial continua, cultivo simple, regado con lluvia, cultivo permanente, por ejemplo, piñas; c) gramíneas, en campo de grande a mediano, distribución espacial continua, cultivo simple, regado con lluvia, cultivo de rotación 0 cambiante, por ejemplo, maíz; d) No gramínea en campo de grande a mediano, distribución continua, al menos dos cultivos adicionales, herbáceos terrestres, simultáneos, regado con lluvia, cultivo de rotación o cambiante, por ejemplo, hortalizas; e) herbácea en campo de grande a mediano, distribución espacial continua, con dos cultivos adicionales, herbáceos terrestres, simultáneos, regado con lluvia, cultivo en barbecho, resultado de una etapa de proceso agrícola.

6) Urbano. Clase de un solo tipo de cobertura: superficie construida, no lineal, área urbana.

7) Suelo desnudo. Clase de un solo tipo de cobertura: superficie no consolidada, suelo desnudo y otro material no consolidado; pedregoso (40-5\%).

\section{Metodología}

La metodología consistió en: la construcción de matrices de tabulación cruzada; el cálculo de persistencia, pérdidas y ganancias; el cálculo de cambios neto e intercambio; el cálculo de cambios totales del paisaje; y el cálculo de transiciones sistemáticas entre categorías (Pontius, Shusas, \& McEachem, 2004), para los períodos antes (1991-1995) y después (2003-2005) de Mitch.

\section{RESULTADOS}

\section{Matriz de Tabulación Cruzada. Persistencia, Pérdidas y Ganancias}

El punto de partida fue la utilización de una imagen de cambio, obtenida a partir de dos imágenes clasificadas de la misma manera por cobertura de la tierra para dos fechas: Fecha-1 la más temprana, Fecha-2 la más tardía, conteniendo ambas las mismas clases. La imagen de cambio tenía asociada una Tabla de Atri- 
butos con información cuantitativa de los cambios detectados entre categorías. En esta Tabla los cambios aparecían expresados en número de pixeles (histograma).

A partir de la Tabla de Atributos se construyó una Matriz de Tabulación Cruzada. En esta matriz, cada fila muestra las categorías de la Fecha-1; cada columna muestra las categorías de la Fecha-2.

Se totalizó el número de pixeles de la matriz, calculando para cada elemento el porcentaje correspondiente. La notación Pij denota la proporción del paisaje que experimenta una transición de la clase $i$ a la clase $j$ donde el número de clases es $J$.

- Las entradas de la diagonal indican la persistencia. Es decir, la proporción del paisaje que persiste en una categoría.

- Las entradas fuera de la diagonal indican la transición a diferentes categorías.

- A la matriz se le agregó una Columna Total. Cada elemento de esta columna (la suma de todos los elementos de la fila) denota la proporción del paisaje en cada una de las categorías en la Fecha-1: Pit.

- Se agregó también una Fila Total. Cada elemento de esta fila (la suma de todos los elementos de la columna) denota la proporción del paisaje en cada categoría en la Fecha-2: Pjt.

El siguiente paso fue examinar las entradas diagonales de la matriz de tabulación cruzada, con los cuales se determinó el porcentaje del paisaje que persistió (persistencia) en cada categoría.

- Después, a la matriz de tabulación cruzada se le agregó una columna y una fila.

- La columna adicional denominada Pérdidas, se calculó a partir del los valores de la Columna Total menos la persistencia de cada categoría. Indica la proporción del paisaje que experimentó una pérdida neta por categoría i, entre Fecha-1 y Fecha-2.

- La fila adicional denominada Ganancias, se calculó a partir de los valores de la Fila Total menos la persistencia. Indica la proporción del paisaje que experi- 
mentó una ganancia neta por categoría j, entre Fecha-1 y Fecha-2.

Con las imágenes de cambio de 1991 y 1995 y de 2003 y 2005 se construyeron dos matrices de tabulación cruzada que se analizaron separadamente para identificar persistencia, ganancias y pérdidas por categorías. En la Tabla 1 y en la Tabla 2 se presentan las Matrices de Tabulación Cruzada de los cambios antes (1991-1995) y después (2003-2006) de Mitch.

Tabla1. Matriz de Tabulación Cruzada para comparar

Mapas de Cobertura de la Tierra de 1991 y 1995.

\begin{tabular}{|c|c|c|c|c|c|c|c|c|c|c|}
\hline & & 1995 & & & & & & & \multirow[b]{3}{*}{$\begin{array}{l}\text { TOTAL } \\
1991\end{array}$} & \multirow[b]{3}{*}{ PERDIDAS } \\
\hline & & 1 & 2 & 3 & 4 & 5 & 6 & 7 & & \\
\hline & & Mixto & Pino & Matorral & Pasto & Agricultura & Urbano & $\begin{array}{r}\text { Suelo } \\
\text { desnudo }\end{array}$ & & \\
\hline \multicolumn{11}{|c|}{1991} \\
\hline 1 & Mixto & 19.24 & 4.51 & 0.17 & 0.00 & 4.35 & 0.00 & 1.04 & 29.31 & 10.08 \\
\hline 2 & Pino & 4.33 & 8.64 & 1.71 & 0.04 & 4.24 & 0.01 & 0.74 & 19.72 & 11.08 \\
\hline 3 & Matorral & 1.21 & 0.93 & 5.04 & 0.44 & 7.75 & 0.03 & 2.49 & 17.90 & 12.86 \\
\hline 4 & Pasto & 0.02 & 0.00 & 1.01 & 0.81 & 0.20 & 0.02 & 0.87 & 2.93 & 2.12 \\
\hline 5 & Agricultura & 3.04 & 0.36 & 2.07 & 0.14 & 10.75 & 0.01 & 3.81 & 20.18 & 9.43 \\
\hline 6 & Urbano & 0.14 & 0.06 & 0.21 & 0.07 & 0.20 & 0.05 & 0.33 & 1.07 & 1.02 \\
\hline 7 & $\begin{array}{l}\text { Suelo } \\
\text { desnudo }\end{array}$ & 1.39 & 0.30 & 1.61 & 0.43 & 2.22 & 0.03 & 2.91 & 8.89 & 5.98 \\
\hline & TOTAL 1995 & 29.36 & 14.80 & 11.82 & 1.94 & 29.73 & 0.16 & 12.19 & 47.43 & 52.57 \\
\hline & GANANCIAS & 10.13 & 6.16 & 6.78 & 1.14 & 18.97 & 0.11 & 9.28 & 52.57 & 100.00 \\
\hline
\end{tabular}

Fuente: Elaboración propia.

Tabla 2. Matriz de Tabulación Cruzada para comparar

Mapas de Cobertura de la Tierra de 2003 y 2006.

\begin{tabular}{|c|c|c|c|c|c|c|c|c|c|c|}
\hline & & 1995 & & & & & & & & \\
\hline & & 1 & 2 & 3 & 4 & 5 & 6 & 7 & \multirow{2}{*}{$\begin{array}{l}\text { TOTAL } \\
1991\end{array}$} & \multirow[b]{2}{*}{ PERDIDAS } \\
\hline & & Mixto & Pino & Matorral & Pasto & Agricultura & Urbano & $\begin{array}{r}\text { Suelo } \\
\text { desnudo }\end{array}$ & & \\
\hline \multicolumn{11}{|c|}{1991} \\
\hline 1 & Mixto & 19.24 & 4.51 & 0.17 & 0.00 & 4.35 & 0.00 & 1.04 & 29.31 & 10.08 \\
\hline 2 & Pino & 4.33 & 8.64 & 1.71 & 0.04 & 4.24 & 0.01 & 0.74 & 19.72 & 11.08 \\
\hline 3 & Matorral & 1.21 & 0.93 & 5.04 & 0.44 & 7.75 & 0.03 & 2.49 & 17.90 & 12.86 \\
\hline 4 & Pasto & 0.02 & 0.00 & 1.01 & 0.81 & 0.20 & 0.02 & 0.87 & 2.93 & 2.12 \\
\hline 5 & Agricultura & 3.04 & 0.36 & 2.07 & 0.14 & 10.75 & 0.01 & 3.81 & 20.18 & 9.43 \\
\hline 6 & Urbano & 0.14 & 0.06 & 0.21 & 0.07 & 0.20 & 0.05 & 0.33 & 1.07 & 1.02 \\
\hline \multirow[t]{3}{*}{7} & $\begin{array}{l}\text { Suelo } \\
\text { desnudo }\end{array}$ & 1.39 & 0.30 & 1.61 & 0.43 & 2.22 & 0.03 & 2.91 & 8.89 & 5.98 \\
\hline & TOTAL 1995 & 29.36 & 14.80 & 11.82 & 1.94 & 29.73 & 0.16 & 12.19 & 47.43 & 52.57 \\
\hline & GANANCIAS & 10.13 & 6.16 & 6.78 & 1.14 & 18.97 & 0.11 & 9.28 & 52.57 & 100.00 \\
\hline
\end{tabular}

Fuente: Elaboración propia. 
Cambio Neto e Intercambio. Cambios Totales del paisaje.

Seguidamente se calculó el intercambio, el cambio neto y el cambio total.

- La cantidad de intercambio (swap) es igual a dos veces el mínimo de la ganancia y pérdidas. Cada celda de la retícula de pixeles que gana es apareada con cada celda que pierde para crear un par de celdas que se intercambian.

- El valor absoluto del cambio neto se calculó a partir del valor máximo de la ganancia y pérdida menos el mínimo de la ganancia y pérdida. Este cambio neto es el resto de no aparear ganancia o pérdida después que todas las ganancias y pérdidas han sido apareadas para calcular el intercambio.

- El cambio total para cada categoría se calculó de la suma del cambio neto y el intercambio, lo que es igual a la suma de las pérdidas y las ganancias.

Agregando los cambios de las categorías individuales se pudo calcular el cambio del paisaje completo. Así:

- El cambio total del paisaje se calculó del total de las ganancias de las categorías individuales, el cual es igual al total de las pérdidas de las categorías individuales. El cambio total en el paisaje es la mitad de la suma de los cambios en las categorías individuales porque los cambios en cada celda cuentan como una ganancia en una categoría y como una pérdida en otra categoría.

- De la misma manera se calculó el intercambio total del paisaje, que es la mitad de la suma de los intercambios en las categorías individuales.

- El total del cambio neto en el paisaje es la mitad de la suma de los cambios netos en las categorías individuales.

En las Tablas 3 y 4 se incluyen los valores de los cambios en términos de porcentajes del paisaje, antes (1991-1995) y después (2003-2005) de Mitch. 
Tabla 3. Valores de los cambios de 1991 a 1995 en términos de porcentaje del paisaje.

\begin{tabular}{|c|l|c|c|c|c|c|}
\hline \multicolumn{2}{|c|}{} & & & & \\
\hline & & & & & \\
GANANCIAS & PERDIDAS & $\begin{array}{c}\text { CAMBIO } \\
\text { TOTAL }\end{array}$ & $\begin{array}{c}\text { CAMBIO } \\
\text { NETO }\end{array}$ \\
\hline 1 & Mixto & 10.13 & 10.08 & 20.20 & 20.15 & 0.05 \\
\hline 2 & Pino & 6.16 & 11.08 & 17.24 & 12.32 & 4.92 \\
\hline 3 & Matorral & 6.78 & 12.86 & 19.64 & 13.57 & 6.07 \\
\hline 4 & Pasto & 1.14 & 2.12 & 3.26 & 2.28 & 0.98 \\
\hline 5 & Agricultura & 18.97 & 9.43 & 28.41 & 18.86 & 9.54 \\
\hline 6 & Urbano & 0.11 & 1.02 & 1.13 & 0.22 & 0.91 \\
\hline 7 & Suelo desnudo & 9.28 & 5.98 & 15.26 & 11.96 & 3.30 \\
\hline & TOTALES & 52.57 & 52.57 & 52.57 & 39.68 & 12.89 \\
\hline
\end{tabular}

Fuente: Elaboración propia.

Tabla 4. Valores de los cambios de 2003 a 2006 en términos de porcentajes del paisaje.

\begin{tabular}{|c|l|c|c|c|c|c|}
\hline & GANANCIAS & PERDIDAS & $\begin{array}{c}\text { CAMBIO } \\
\text { TOTAL }\end{array}$ & INTERCAMBIO & $\begin{array}{c}\text { CAMBIO } \\
\text { NETO }\end{array}$ \\
\hline 1 & Mixto & 4.11 & 7.24 & 11.35 & 8.22 & 3.13 \\
\hline 2 & Pino & 9.27 & 7.65 & 16.92 & 15.30 & 1.62 \\
\hline 3 & Matorral & 12.27 & 6.74 & 19.01 & 13.47 & 5.54 \\
\hline 4 & Pasto & 1.01 & 5.40 & 6.41 & 2.02 & 4.39 \\
\hline 5 & Agricultura & 14.63 & 11.99 & 26.62 & 23.98 & 2.64 \\
\hline 6 & Urbano & 0.10 & 0.12 & 0.22 & 0.20 & 0.02 \\
\hline 7 & Suelo desnudo & 7.39 & 9.64 & 17.03 & 14.77 & 2.25 \\
\hline & TOTALES & 48.78 & 48.78 & $\mathbf{4 8 . 7 8}$ & 38.98 & 9.80 \\
\hline
\end{tabular}

Fuente: Elaboración propia.

Los resultados anteriores ponen de manifiesto que entre 1991-1995 el mayor cambio neto fue en agricultura (9.54\%), matorral $(6.07 \%)$ y pino $(4.92 \%)$. Para $2003-2006$, en matorral $(5.54 \%)$, pasto $(4.39 \%)$ y bosque mixto $(3.13 \%)$.

Que entre 1991-1995 el intercambio se dio en la mayoría de las categorías: bosque mixto $(20.15 \%)$, agricultura $(18.86 \%)$, matorral $(13.56 \%)$, pino $(12.32 \%)$ y 
suelo desnudo (11.96\%); y entre 2003-2006 en: agricultura (23.98\%), pino (15.30\%), suelo desnudo (14.77\%), matorral (13.47) y bosque mixto (8.22\%).

Los resultados evidencian una alta dinámica de cambio del paisaje, $52.57 \%$ entre 1991- 1995 y $48.78 \%$ entre 2003-2006.

\section{TRANSICIONES SISTEMÁTICAS ENTRE CATEGORÍAS}

\section{Análisis de Ganancias y Pérdidas}

El paso siguiente fue examinar las entradas fuera de la diagonal de la matriz de tabulación cruzada para identificar las transiciones sistemáticas más prominentes entre la Fecha-1 y la Fecha-2. Para ello, a partir de esta matriz se construyeron dos tablas de transiciones, una para analizar las ganancias, otra para analizar las pérdidas.

Tabla para análisis del porcentaje de cambio de la cobertura de la tierra en términos de ganancias.

La tabla para análisis de ganancias se construyó manteniendo los valores de la matriz de tabulación cruzada, agregando intercaladamente tres filas de manera que para cada categoría se tuvieran cuatro filas.

Fila 1. En negritas. Representa el porcentaje observado combinado en el paisaje: $P i j$. Igual a la matriz de tabulación cruzada.

Fila 2. Cursiva. Representa el porcentaje combinado que se esperaría si la ganancia en cada categoría ocurriera al azar. Se calculó a partir de:

$$
G_{i j}=\left(P_{+j}-P_{j j}\right)\left(\frac{P_{l+}}{\sum_{i=1, i \neq j}^{\prime} P_{l+}}\right) \quad(\text { Ecuación 1) }
$$

donde para la ganancia Gij: i es la categoría de Fecha-1, j es la categoría de Fecha-2; $J$ es el número total de categorías; Pit es la proporción del paisaje en la categoría i en la Fecha-1; $P+j$ es la proporción del paisaje en la categoría $j$ en Fecha-2. Para las entradas de la diagonal, el número esperado es igual al número observado de manera que la matriz resultante de las transiciones aleatorias tiene la misma cantidad de persistencia que el porcentaje observado. 
Fila 3. (Paréntesis curvo). Es la combinación de la proporción observada menos la proporción esperada bajo un proceso aleatorio. En otras palabras, el valor de la Fila 1 menos el valor de la Fila 2: (Pij - Gij).

Fila 4. [Paréntesis angular]. Es la combinación de la diferencia relativa entre el número observado y el número esperado. En otras palabras, el valor de la Fila 1 dividido por el valor de la Fila 2: (Pij-Gij)/Gij.

Tabla para análisis del porcentaje de cambio de la cobertura de la tierra en términos de pérdidas.

La lógica de construcción de esta tabla fue la misma que la de la tabla anterior solo que el papel de las filas y las columnas cambió.

Fila 1. En negritas. Es la combinación del porcentaje observador sobre el paisaje. Es lo mismo de la tabla anterior.

Fila 2. Cursiva. Es la combinación del porcentaje que sería esperado si las pérdidas en cada categoría hubiesen ocurrido al azar. Se calculó a partir de:

$$
L_{i j}=\left(P_{i+}-P_{i i}\right)\left(\frac{P_{+j}}{\sum_{j=1, j \neq i}^{J} P_{+j}}\right) \quad(\text { Ecuación 2) }
$$

Para la pérdida Lij. En este caso también, el número esperado es igual al número observado para las entradas de la diagonal con el objeto de mantener constante el nivel de persistencia sobre el paisaje observado.

Fila 3. (Paréntesis curvo). Es la combinación de la proporción observada menos la proporción esperada bajo un proceso aleatorio. Se calculó a partir de: (Pij - Lij).

Fila 4. [Paréntesis angular]. Como en la Tabla anterior, la magnitud del número indica la diferencia entre el valor observador y el valor esperado, relativo a la magnitud del valor esperado.

Se calculó a partir de: $(P i j-L i j) / L i j$.

En las Tablas 5.A y 5.B y en las Tablas 6.A y 6.B se presentan los Análisis de las Ganancias seguidos de las Transiciones más Sistemáticas entre categorías 
para 1991-1995 y para 2003-2006 respectivamente, antes y después de Mitch.

Tabla 5.A - Análisis de Ganancias 1991-1995.

\begin{tabular}{|c|c|c|c|c|c|c|c|c|c|}
\hline & 1995 & & & & & & & & \\
\hline & & 2 & 3 & 4 & 5 & 6. & & Fotal 1601 & peragons \\
\hline & Wuits & Sing & Menarral & 2uts & Aricultura & Jrbang & Syelo denuda & & \\
\hline 199. & & & & & & & & & \\
\hline 1 Mluso & 19.24 & 4.51 & 0.17 & 0.00 & 4.35 & 0.00 & 1.04 & 23.51 & 10.00 \\
\hline & 29.24 & 2.25 & 2.42 & 0.54 & 6.97 & o.as & 280 & 34.24 & 15.00 \\
\hline & 0.00 & 226 & 225 & 0.34 & -262 & -0.03 & -195 & 4.92 & -4.92 \\
\hline & 0.00 & 101 & 0.92 & 0.99 & -0.38 & -0.58 & -0.65 & 0.14 & -0.33 \\
\hline 2 Pino & 4.33 & 8.64 & 1.71 & 0.04 & 4.24 & 0.01 & 0.79 & 19.72 & $11.6 \mathrm{~B}$ \\
\hline & 2.52 & 8.64 & 1.63 & 0.25 & 4.65 & 0.02 & $20:$ & 2004 & 12.40 \\
\hline & 150 & 0.00 & 0,05 & 0.19 & -0.44 & -0.01 & -127 & 0.32 & -0.32 \\
\hline & 0.53 & 000 & 0.05 & -0.82 & -0.09 & -0.45 & -0.65 & -0.02 & -0.03 \\
\hline 8Matoral & 1.21 & 0.93 & 5.04 & 0,44 & 7.75 & 0.63 & 2.49 & 17,50 & 12.85 \\
\hline & 2.56 & 1.37 & 5.06 & 0.22 & 4.25 & 0.02 & 1.82 & 15.28 & 1025 \\
\hline & -1.36 & 0.45 & 0.00 & 0.23 & 3.50 & 0.01 & 0.57 & 261 & 2.61 \\
\hline & .0 .58 & .0 .32 & 0.00 & 1.12 & 0.82 & 0,0 & 0.37 & 0.17 & 0.28 \\
\hline aperto & 0,02 & 0.00 & 1,01 & 0.81 & 0.20 & 0.62 & 6.87 & 2,93 & 2.12 \\
\hline & 0.42 & 0.22 & 0.24 & 0.02 & 0.70 & 0.00 & 0.50 & 2.69 & 100 \\
\hline & -0.48 & .0 .22 & का & 0.00 & -0.49 & 0.02 & 0.57 & 0.24 & 0.24 \\
\hline & .0 .98 & .0 .98 & 317 & 0.00 & -0.01 & $3 \pi$ & 1.90 & 0.00 & 0.13 \\
\hline 5farriculara & 3.04 & 0.36 & 2.07 & 0.14 & 10.75 & 0.01 & 3.81 & 20.18 & 2.43 \\
\hline & 2.85 & 1.55 & 1.67) & 0.24 & 10.75 & 0.02 & 200 & 29.17 & 5.42 \\
\hline & 0.19 & .110 & 0.40 & .0 .00 & 0.00 & -0.01 & 1.75 & 101 & 1.01 \\
\hline & 0.05 . & -0.77 & 0.24 & 0.9 & 0,0 & -0.59 & 0.85 & 0.05 & 0.12 \\
\hline 6 urbano & 0.14 & 0.06 & 0.21 & 0.07 & 0.20 & 0.05 & 0.33 & 1.07 & 102 \\
\hline & 0.15 & 0.68 & 0.09 & 0.02 & 0.25 & 0.05 & 0.11 & 0.75 & 0.70 \\
\hline & -0.01 & -0.02 & 0.13 & 0.06 & -0.05 & 0,0 & 0.22 & 0.32 & 0.32 \\
\hline & 0.09 & .0 .79 & 147 & 4.93 & -0.19 & 0,0 & 200 & 0.43 & 0.46 \\
\hline 7 şualodennuda & 1.38 & 0.30 & 1.61 & 0.43 & 2.22 & 0.03 & 2.91 & 1.00 & 5.9. \\
\hline & 1.27 & 0.68 & 0.73 & 0.10 & 2.11 & 0.01 & 294 & 7.83 & 492 \\
\hline & 0.12 & 0.38 & 0.87 & 0.33 & 0.11 & 0.02 & 0.00 & 106 & 1.05 \\
\hline & 0,05 & 0.56 & 1.15 & 312 & 0.05 & 2.27 & 0.00 & 0.14 & 0.22 \\
\hline FOTAL 1995 & 29,36 & 14.80 & 11,82 & 1,94 & 29.73 & 0.16 & 12.19 & 100,00 & 52.57 \\
\hline & 29.36 & 14.80 & 11.82 & 1.94 & 29.73 & 0.16 & 12.15 & 160.00 & 52,57 \\
\hline & 0.00 & 000 & 0.00 & 000 & 000 & 0,0 & 0.00 & 0,0 & 0.00 \\
\hline & 0.00 & 0.00 & 0.00 & 0.00 & 0.00 & 0.00 & 0.00 & 0,00 & 0.00 \\
\hline GARLAKIAS & 10.13 & 6.16 & 6.78 & 1.14 & 18,97 & 0.11 & 9.28 & 52.57 & \\
\hline & 20.13 & 6.26 & 6.78 & 1.14 & 18.97 & 0.11 & 928 & 52.57 & \\
\hline & 0.00 & 0.00 & 0.00 & 000 & 0.00 & 000 & 0.00 & 0.00 & \\
\hline & 0.00 & 0.00 & 0.00 & 0.00 & 0.00 & 0.00 & 0.00 & 0.00 & \\
\hline
\end{tabular}

Fuente: Elaboración propia. 
Tabla 5.B - Transiciones más sistemáticas entre categorías 1991-1995.

\section{GANANCIAS}

\begin{tabular}{|c|c|c|c|}
\hline Transidion & $\begin{array}{l}\text { (Obaervada } \\
\text { menos } \\
\text { esperada] }\end{array}$ & $\begin{array}{l}\text { [Diferencia } \\
\text { dividida } \\
\text { por } \\
\text { esperada] }\end{array}$ & Interpretación de transición sistemśtics \\
\hline Pineen 1991 y Boscue mixto en 1995 & 1.50 & 053 & $\begin{array}{l}\text { Cuando bostgas mixto eanó, reemplaź́a } \\
\text { pino. }\end{array}$ \\
\hline $\begin{array}{l}\text { Matorral en } 1991 \text { y Bosque mixto en } \\
1995\end{array}$ & -1.36 & -0.53 & $\begin{array}{l}\text { Cuando bosęue minto ganó, no reemplazo a } \\
\text { matorral. }\end{array}$ \\
\hline Bosque mixto en 1991 y Pine en 1995 & 2.26 & 1.01 & $\begin{array}{l}\text { Cuando pino ganó, reemplaco a bosque } \\
\text { mixto. }\end{array}$ \\
\hline Agricultura en 1991 y Pino en 1995 & -1.19 & -0.71 & $\begin{array}{l}\text { Cuando pino ganó, no reemplazs a } \\
\text { sariculturd. }\end{array}$ \\
\hline $\begin{array}{l}\text { Bosque mixto en } 1991 \text { y Maromal en } \\
1995\end{array}$ & -225 & -0.93 & $\begin{array}{l}\text { Cuando matorral gano, no reemplazo a } \\
\text { Bosque mixto. }\end{array}$ \\
\hline Pastoen 1991 y Matorral en 1995 & 0.37 & 3.17 & Cuando matorral ganó, reemplazó a pasto. \\
\hline $\begin{array}{l}\text { Bosque mixto en } 1991 \text { y Agricultura en } \\
1095\end{array}$ & -2.62 & -0.38 & $\begin{array}{l}\text { Cuando agricultura gono, no reemplazóa } \\
\text { besque mixto. }\end{array}$ \\
\hline Matorral en 1991 y Agricultura en 1995 & 3.50 & 0.82 & $\begin{array}{l}\text { Cuando agricultura ganó, reemplazo a } \\
\text { matorral. }\end{array}$ \\
\hline $\begin{array}{l}\text { Ausque mixto en } 1991 \text { y No Bosque } \\
\text { Mixto en } 1996\end{array}$ & -4.92 & -0.33 & $\begin{array}{l}\text { Cuanda Categorias No Bosque Mato } \\
\text { ganaren, ellas no reemplazaron a Bosque } \\
\text { miato. El Bosque mato no perdiá. }\end{array}$ \\
\hline $\begin{array}{l}\text { Matorralen } 1991 \text { y No Matorral en } \\
1995 .\end{array}$ & 2.61 & 0.25 & $\begin{array}{l}\text { Cuando Categorias No Matorral ganaron, } \\
\text { ellas reemplazaron a Matorral. Matorral } \\
\text { perdic. }\end{array}$ \\
\hline
\end{tabular}

Fuente: Elaboración propia.

De los resultados presentados en la Tabla 5.B interpretamos como las transiciones más sistemáticas en ganancia:

- Una regeneración de Bosque mixto a partir de Pino.

- El Pino ganó a partir de pérdida de Bosque mixto.

- La Agricultura en barbecho. Matorrales sustituyen a Pastos.

- Cultivos sustituyen a Matorrales.

- El Pino sustituye a Matorral. Agricultura sustituye a Matorral. 
Tabla 6.A - Análisis de Ganancias 2003-2006.

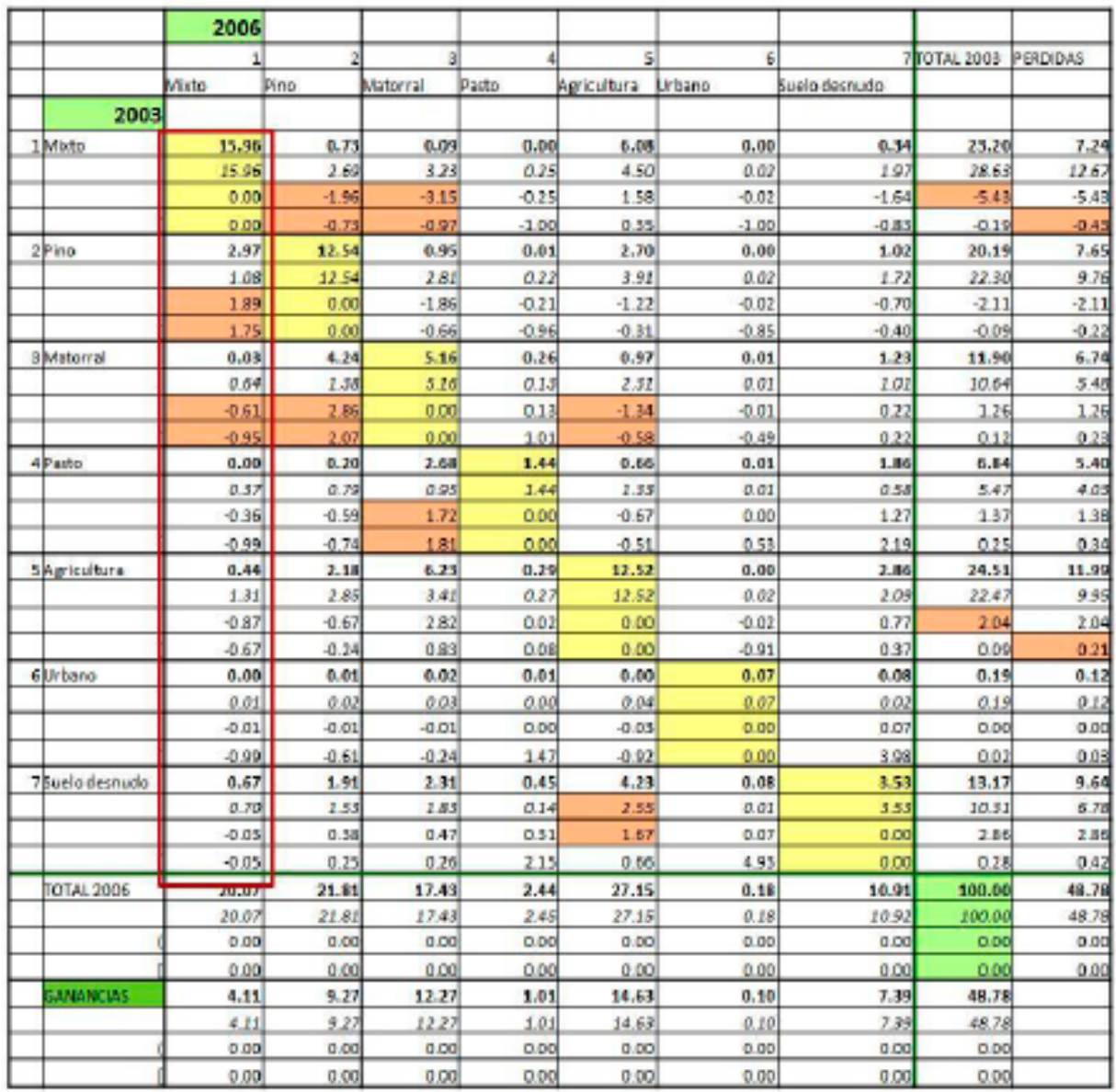

Fuente: Elaboración propia. 
Tabla 6.B - Transiciones más sistemáticas entre categorías 2003-2006.

\begin{tabular}{|c|c|c|c|}
\hline Tranuldín & $\begin{array}{l}\text { (Ohaerva- } \\
\text { da meness } \\
\text { esperada) }\end{array}$ & $\begin{array}{l}\text { [Diferventia } \\
\text { dividida } \\
\text { par } \\
\text { esperada] }\end{array}$ & Inturprutacián de trantidib́n sintembitica \\
\hline Proen 2003 y Besgue Misto en 2006 & 1.89 & 175 & $\begin{array}{l}\text { Cuande Basque misto ganó, reerplazé a } \\
\text { Pino. }\end{array}$ \\
\hline $\begin{array}{l}\text { Matorral en } 2003 \text { y Bosque Miato en } \\
2006 .\end{array}$ & -0.61 & -0.95 & $\begin{array}{l}\text { Cuando Bosque miato gand, no } \\
\text { rocmplazo a Matorral. }\end{array}$ \\
\hline Basque minta en 2003 y Pina en 2006 & -1.96 & -0.73 & $\begin{array}{l}\text { Cuande Fine gand, no revmplaź a } \\
\text { Boscowe mixte. }\end{array}$ \\
\hline Matorral en 2003y yino en 2006 & 2.96 & 207 & Cuando fine geno, reemplazbal Matorral \\
\hline $\begin{array}{l}\text { Basque mirta en } 2003 \text { y Matorral en } \\
2006 .\end{array}$ & -3.15 & -0.97 & $\begin{array}{l}\text { Cuando Material gané, no reemplaźa a } \\
\text { Bescque Mixto. }\end{array}$ \\
\hline Pasto en 2003 y Matorral en 2006 & 1.72 & 1.81 & $\begin{array}{l}\text { Cuando Materral gand, remplazo al } \\
\text { Pasto. }\end{array}$ \\
\hline Matorral en 2003 y Aericultura en 2006 & -1.34 & -0.58 & $\begin{array}{l}\text { Cuande Ag gicultute ganó, ne reemplazóa } \\
\text { Matorral. }\end{array}$ \\
\hline $\begin{array}{l}\text { uelo desnude en } 2003 \text { y Aericultursen } \\
2006\end{array}$ & 2.55 & 167 & $\begin{array}{l}\text { Cuande Agriculture ganó, reemplacós. } \\
\text { puelo desnude. }\end{array}$ \\
\hline $\begin{array}{l}\text { Basque miato en } 2003 \text { y No Bosque } \\
\text { mixto en } 2006\end{array}$ & $-5,43$ & -0.43 & $\begin{array}{l}\text { Cuande las Catequrias de No Basque } \\
\text { miato ganaran, ellas no reemplacaron a } \\
\text { Besque mixto. Bosque mixto no perdió. }\end{array}$ \\
\hline $\begin{array}{l}\text { Agricultura en } 2003 \text { y No Agricultura en } \\
2006\end{array}$ & 2.04 & 0.21 & $\begin{array}{l}\text { Cuande las Catogorlas de No Aggicultura } \\
\text { ganaron, elas resmplazaron a Agrioultura } \\
\text { Agrioultura perdic. }\end{array}$ \\
\hline
\end{tabular}

Fuente: Elaboración propia.

De los resultados presentados en la Tabla 6.B interpretamos como las transiciones más sistemáticas en ganancia:

- Regeneración del Bosque mixto a partir del Pino.

- Regeneración de Pino a partir de Matorral.

- Regeneración de Matorral a partir de Pasto (Agricultura).

- Agricultura surge de un suelo preparado.

- El Bosque mixto se mantuvo.

En las Tablas 7.A y 7.B y en las Tablas 8.A y 8.B se presentan los Análisis de las Pérdidas seguidos de las Transiciones más Sistemáticas entre categorías para 1991-1995 y para 2003-2006 respectivamente, antes y después de Mitch. 
Tabla 7.A -. Análisis de Pérdidas 1991-1995

\begin{tabular}{|c|c|c|c|c|c|c|c|c|c|c|}
\hline & & 1995 & & & & & & & & \\
\hline & & 1 & 2 & y. & 4 & s. & 6 & & DTAL 1601 & PESODAS \\
\hline & & Nato & Pho & Astornal & Porto & Agrioubura & Jirbse & such desudo & & \\
\hline \multicolumn{11}{|c|}{1991} \\
\hline & Hawo & 19.24 & 4,51 & 0.17 & 0.00 & 4.35 & 0.60 & 1,14 & 29.31 & 11.08 \\
\hline & & 29.24 & 2.11 & 1.60 & 0.28 & 4.24 & a.az & 1.74 & 2931 & $10.0 \mathrm{~S}$ \\
\hline & & 0.00 & 240 & -152 & -0.27 & a.11 & -0.02 & 0.00 & $O D O$ & 0.00 \\
\hline & & 0.00 & 114 & -0.80 & 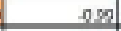 & 003 & -0.97 & 2.04 & 0.0 & 0,0 \\
\hline \multirow{4}{*}{\multicolumn{2}{|c|}{$2 p$ ho }} & 4.53 & 1.64 & $1 . \pi$ & 0.04 & 4.24 & 1.01 & 0.14 & 12.72 & 11.00 \\
\hline & & 3.82 & 8.64 & 1.56 & $0>5$ & 3.87 & 0,02 & 150 & 1472 & $11.0 \mathrm{~s}$ \\
\hline & & 0.51 & 0.00 & 0.16 & -024 & 038 & -0.01 & -0.34 & 0.00 & 0,0 \\
\hline & & 0.13 & 0.0 & 0.12. & -0.83 & 0.10 & -0.41 & 0.5. & 000 & 0.00 \\
\hline \multirow{4}{*}{\multicolumn{2}{|c|}{8 Astorial }} & 1.21 & 0,93 & 5.64 & 0.44 & 7,75 & 3.03 & 2,49 & 17,50 & 12,66 \\
\hline & & $4.2 \mathrm{~s}$ & 2.16 & 5.04 & 0.28 & 433 & 0.02 & 1.7 & 1790 & 12.ed \\
\hline & & .3 .07 & .123 & 0.00 & 0.16 & 342 & 0.01 & 0.72 & 0,0 & 0.00 \\
\hline & & .0 .72 & .057 & 0.00 & 0.57 & arg & 0.47 & 0.40 & 0.00 & 0.00 \\
\hline \multirow{4}{*}{\multicolumn{2}{|c|}{ ¿patoa }} & 0.02 & 0.00 & 1.01 & 0.41 & 0.20 & 0.02 & 0.18 & 2.03 & 2.12 \\
\hline & & 0.64 & 0.32 & 0.25 & 0.81 & 064 & 0.00 & 0.26 & 293 & 2.12 \\
\hline & & 0.62 & 0.32 & 0.73 & 0.00 & -0.44 & 0.02 & asd & 0.00 & 0.00 \\
\hline & & -0.97 & 10,4 & 2.4 & 0,0 & -068 & 5.48 & 2.28 & 0,0 & 0.0. \\
\hline \multirow{4}{*}{\multicolumn{2}{|c|}{ sqikuturs }} & 3.04 & 0.96 & 2.00 & 0.14 & $10 \pi 5$ & 3.01 & 3.11 & 20.13 & 3.43 \\
\hline & & 3.94 & 1.98 & $1.5 \mathrm{~g}$ & $0 x$ & 1075 & 0.02 & 1.54 & 2019 & 2.45 \\
\hline & & -0.90 & -163 & Q.48 & -0.12 & 000 & -0.04 & 2.17 & 000 & 0.00 \\
\hline & & .0 .23 & .0 .22 & 0.50 & -0.45 & $a 00$ & -0.56 & 138 & 0.00 & 0.00 \\
\hline \multirow{4}{*}{\multicolumn{2}{|c|}{ Gurbons }} & 0.14 & 0.66 & 0.21 & 0.07 & a.20 & 3.06 & 0,33 & 107 & 1.02 \\
\hline & & 0.30 & 0.15 & 0.12 & 0.02 & 030 & 0.05 & 0.28 & 107 & 1.02 \\
\hline & & .0 .16 & 0.00 & 0.09 & O.DS & -0.10 & 0.00 & 0.21 & 0,0 & 0.00 \\
\hline & & 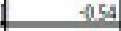 & -0.61 & 0.25 & .279 & -0.33 & 0.0 & 168 & 0.0 & 0.0. \\
\hline \multirow{4}{*}{\multicolumn{2}{|c|}{ Tuelodennuds }} & 1.39 & 0.90 & 1.61 & 0.43 & 2.22 & 0.03 & 2.31 & L.p & $5 . \mathrm{ma}$ \\
\hline & & 2.00 & 1.02 & 0.61 & 0.13 & 202 & 0.02 & 2.31 & $\Delta .69$ & 5.95 \\
\hline & & 0.61 & 0.71 & 0.60 & 0.30 & 020 & 0.02 & 0.09 & 000 & 0.00 \\
\hline & & .0 .30 & -0.70 & 100 & 2.35 & 0.10 & 2.02 & oud & 0,00 & 0.00 \\
\hline \multirow{4}{*}{\multicolumn{2}{|c|}{ TOTALIS95 }} & 29,36 & $14.60)$ & $11, \mathrm{k}$ & 1.94 & 29.73 & 0.16 & 12,15 & 10060 & 52,57 \\
\hline & & 34.31 & $\operatorname{tans}$ & 21.05 & 2.00 & 20.10 & 0.18 & 20.94 & 10000 & $52.5 \mathrm{X}$ \\
\hline & & -4.25 & -1.57 & $0 . \pi$ & -0.00 & 350 & 0.01 & 2.5 & $0 D 0$ & 0.00 \\
\hline & & 4.14 & 20.10 & 0.00 & 0.04 & 0.14 & 0.0 & 0.27 & 0,0 & 0,0 \\
\hline \multirow{4}{*}{\multicolumn{2}{|c|}{ GANANDAS }} & 10.13 & 0.16 & $0 . \pi$ & 1.14 & 11.97 & 0.11 & 9.21 & 52.57 & \\
\hline & & 14.98 & 2.73 & 5.98 & 123 & 564 & 0.10 & 2.13 & 5257 & \\
\hline & & .4 .65 & -157 & 0.75 & -0.09 & 356 & 0.01 & 2.19 & 0.00 & \\
\hline & & 0.32 & -0.20 & 0.13. & -0.07 & 0.23 & 0,0 & $0.3 d$ & 0.0 & \\
\hline
\end{tabular}

Fuente: Elaboración propia. 
Tabla 7.B - Transiciones más sistemáticas entre categorías 1991-1995.

PERDIDAS

\begin{tabular}{|c|c|c|c|}
\hline Tramdicilen & $\begin{array}{l}\text { (observado } \\
\text { menes } \\
\text { esperado) }\end{array}$ & $\begin{array}{l}\text { [Dilerencia } \\
\text { elluidida } \\
\text { por } \\
\text { esparado] }\end{array}$ & Interpetatacián de transicion sistemática \\
\hline Dosque mixto en 1991 y Pino en 1995. & 240 & 114 & $\begin{array}{l}\text { Cuando Bosque mixto perdio, lo reemplaxo } \\
\text { Pino. }\end{array}$ \\
\hline $\begin{array}{l}\text { Bosque miato en } 1991 \text { y Matorral en } \\
1995 .\end{array}$ & -1.52 & -0.50 & $\begin{array}{l}\text { Cuando Blosque miato perdią, no la reomplazd } \\
\text { Matorral. }\end{array}$ \\
\hline Pino en 1991 y Aaricultura en 1995 & 0.38 & a.10 & Cuando Pino perdio, lo reemplazósaricultura. \\
\hline Fino en 1991 y Sudio dernudo en 1995. & -0.84 & -0.53 & $\begin{array}{l}\text { Cuando Fino perdio, no lo reemplazo Suclo } \\
\text { desnudo. }\end{array}$ \\
\hline Matorralen 1991 y Pin o en 1995 & -1.23 & 057 & $\begin{array}{l}\text { Cusndo Matorrad perdió, no lo reemplazo el } \\
\text { Pino. }\end{array}$ \\
\hline Matorralen 1991 y Agricultura en 1995 & 3.42 & $a .79$ & $\begin{array}{l}\text { Cuando Matorral perdis, lo reemplaxo } \\
\text { Agricultura. }\end{array}$ \\
\hline Agricultura en 1991 y Pina en 1995 & -1.63 & -0.82 & $\begin{array}{l}\text { Cuando Agriculture perdis, no bo reempleab ol } \\
\text { Pina. }\end{array}$ \\
\hline $\begin{array}{l}\text { Agricultura en } 1991 \text { y Suelo derrudo en } \\
1995\end{array}$ & 2.17 & 133 & $\begin{array}{l}\text { Cuando Agricultura perdio, lo reemplazó el } \\
\text { puelo desnudo. }\end{array}$ \\
\hline $\begin{array}{l}\text { No Basque mixte en } 1991 \text { y Basque } \\
\text { misto en } 1995\end{array}$ & -4.84 & -0.32 & $\begin{array}{l}\text { Cuando las Categorias No Basque mizto } \\
\text { perdieron, Categerias de No Bosque las } \\
\text { reemplazaron. Bonque mixto ne gand. }\end{array}$ \\
\hline $\begin{array}{l}\text { No Agricultura en } 1991 \text { y Agricultura en } \\
\text { 1005 }\end{array}$ & 3.57 & 0.23 & $\begin{array}{l}\text { Cuando Categorias No Agricultura perdioran, } \\
\text { Agricultura las reomplaob. Apricultura gano. }\end{array}$ \\
\hline
\end{tabular}

Fuente: Elaboración propia.

De los resultados presentados en la Tabla 7.B interpretamos como las transiciones más sistemáticas en pérdidas:

- Ganó Pino a partir de pérdida de Bosque mixto.

- Agricultura hace perder a Pino.

- Cultivos sustituyen a Matorrales.

- Al terminar un cultivo lo sustituye suelo desnudo.

- Agricultura sustituye a las otras categorías. 
Tabla 8. A. - Análisis de Pérdidas 2003-2006

\begin{tabular}{|c|c|c|c|c|c|c|c|c|c|c|}
\hline & & 2006 & & & & & & & & \\
\hline & & 1 & 2 & 3 & 4 & 5) & 6. & & FOTAL 2003 & PEADEDAS \\
\hline & & Misto & Pine & Maborral & Paste & sarigulture & Urbane & Suslo desnudo & & \\
\hline & 2003 & & & & & & & & & \\
\hline & Muto & 15.96 & 0,73 & 0.09 & 0.00 & 6.08 & 0.00 & 0.34 & 23,20 & 7.20 \\
\hline & & 1596 & 2.98 & 1.53 & 0.22 & 2.46 & 002 & 0.99 & 23.20 & 7.24 \\
\hline & & 0.00 & -1.24 & -1.49 & -0.22 & 3.62 & -0.02 & -0.65 & 0.00 & 0.00 \\
\hline & & 0.00 & -0.63 & -0.95 & 1.00 & 147 & -100 & -0.68 & 0.00 & 0.00 \\
\hline & Pino & 2.97 & 12,54 & 0.95 & 0.01 & 2,70 & 0.00 & 1.02 & 20,19 & 7.65 \\
\hline & & 196 & 12.54 & 171 & 0.24 & 2.66 & 0.02 & 2.07 & 20.19 & 7.65 \\
\hline & & 100 & 0.00 & -0.75 & -0.23 & 0.04 & -0.01 & -0.05 & 0.00 & 0.00 \\
\hline & & 0.51 & 0.00 & -0.44 & -0.97 & 0.01 & -0.82 & -0.04 & 0.00 & 0.00 \\
\hline 3 & Matorral & 0.03 & 4.24 & 5.16 & 0.26 & 0.97 & 0.01 & 1.23 & 11.90 & 6.74 \\
\hline & & 1.64 & 1.78 & 5.16 & 0.20 & 2.22 & 0.01 & 0.89 & 11.90 & 6.24 \\
\hline & & -101 & 245 & 000 & 0.05 & $-1.2 \%$ & $-0,01$ & 0.54 & 0.00 & 000 \\
\hline & & -3.24 & 1.38 & 0.00 & -0.14 & -0.56 & -0.57 & -0.55 & 0.00 & 0.00 \\
\hline & Pasto & 0.00 & 0.20 & 2.68 & 1.44 & 0.66 & $0.0 \mathrm{t}$ & $1.8 \mathrm{E}$ & 6.84 & 5.40 \\
\hline & & 1.13 & 2.21 & 097 & 1.44 & 2.50 & 003 & 0.68 & 6.34 & $5.4 d$ \\
\hline & & -1.11 & -1.00 & 171 & 0.00 & -0.58 & $0 \infty$ & 1.28 & 0.00 & 0,0 \\
\hline & & -1.00 & -0.83 & 1.77 & 0.00 & -0.56 & 0.00 & 203 & 0.00 & 0.00 \\
\hline & Mriculara & 0.44 & 2.18 & 6.23 & 0.20 & 12.52 & 0.00 & 2.86 & 24.51 & 11.99 \\
\hline & & 330 & 3.59 & $28 \pi$ & 0.40 & 12.52 & 003 & 2.80 & 24.51 & 12.99 \\
\hline & & -2.87 & -1.41 & 3.36 & -0.12 & 0.00 & -0.03 & 1.08 & 0.00 & 0.00 \\
\hline & & -0.97 & -0.36 & 1.17 & -0.20 & 0.00 & .0 .93 & 0.50 & 0.00 & 0.00 \\
\hline & Jibono & 0.00 & 0.01 & 0.02 & 0.01 & 0.00 & 0.07 & 0,08 & 0.19 & 0,12 \\
\hline & & 0.02 & 0.03 & 0.02 & 0.00 & 0.03 & 0.07 & 0.01 & 0.19 & 0.22 \\
\hline & & -0.02 & -0.02 & 0.00 & 0.00 & -0.03 & 0.00 & 0.07 & 0.00 & 0.00 \\
\hline & & -0.99 & $-0.5 \mathrm{~T}$ & -0.02 & 0.75 & -0.91 & 0.00 & 5.28 & 0.00 & 0.00 \\
\hline & Suelo dernudo & 0.67 & 1.91 & 2.31 & 0.45 & 4.23 & 0.08 & 3.53 & 13.17 & 9.64 \\
\hline & & 2.17 & 2.36 & 1.59 & 0.26 & 2.94 & 0.02 & 3.53 & 23.17 & 2.64 \\
\hline & & -150 & -0.45 & 0.42 & 0.18 & 1.25 & 0.06 & 0.00 & 0.00 & 0.00 \\
\hline & & -0.09 & -0.19 & 022 & 0.70 & 044 & 3.15 & 0.08 & 0.00 & 0,00 \\
\hline & TOTAL 2006 & 20.07 & 21.81 & 17.43 & 2.44 & 27.15 & 0.18 & 10.91 & 100.06 & $48.7 \mathrm{E}$ \\
\hline & & 2618 & 23.48 & 14.19 & 2.77 & 24.39 & 0.18 & 8.89 & 100.00 & 48.78 \\
\hline & & -6.10 & -1.67 & 3.25 & -0.32 & 2.83 & 0.00 & 203 & 0.00 & 0.00 \\
\hline & & -0.23 & -0.07 & 0.23 & -0.12 & 0.12 & -0.02 & 0.28 & 0.00 & 0.00 \\
\hline & EARAANCIAS & 4.11 & $0.2 \mathrm{~J}$ & 12.27 & 1.01 & 14.53 & 0.10 & 7.38 & 4E.TE & \\
\hline & & 10.21 & 20.94 & 2.03 & 1.33 & 12.81 & 0.11 & 5.38 & 43.78 & \\
\hline & & -6.10 & -1.67 & 3.25 & -0.32 & 2.83 & 0.00 & 2.03 & 0.00 & \\
\hline & & -0.60 & -0.15 & 0.36 & -0.24 & 024 & $-0,04$ & 0.58 & 0.00 & \\
\hline
\end{tabular}

Fuente: Elaboración propia. 
Tabla 8.B - Transiciones más sistemáticas entre categorías 2003-2006.

PERDIDAS

\begin{tabular}{|c|c|c|c|}
\hline Transicion & $\begin{array}{l}\text { [Observado } \\
\text { menas } \\
\text { emperado] }\end{array}$ & $\begin{array}{l}\text { [Diferendia } \\
\text { dividida } \\
\text { por } \\
\text { esperado] }\end{array}$ & Interpretacion de transicion sibtematica \\
\hline Bosque mixto en 2003 y Materral en 2006 & -1.49 & -0.95 & $\begin{array}{l}\text { Cuarndo Borkque mixto perdic, no lo } \\
\text { roomplaca Matornal. }\end{array}$ \\
\hline $\begin{array}{l}\text { Bosique misto en } 2003 \text { y Agricultura en } \\
2005\end{array}$ & 3.62 & 1.47 & $\begin{array}{l}\text { Cuando Bosque mixto pondic, lo } \\
\text { reemplaco Agricultura. }\end{array}$ \\
\hline Pino en 2003 y Bosque mixto en 2006 & 1.00 & 0.51 & $\begin{array}{l}\text { Cuando Pino perdiô, lo reemplazo } \\
\text { Bosque mixte. }\end{array}$ \\
\hline Pino en 2003 y Matorral en 2000 & -0.35 & -0.44 & $\begin{array}{l}\text { Guando Fino perdio, no lo reemplazo } \\
\text { Matorral. }\end{array}$ \\
\hline Matorral en 2003 y Bosque mixto en 2006 & -1.61 & -3.26 & $\begin{array}{l}\text { Cusndo Mstorral perdib, no lo reemplazó } \\
\text { Basque mixto. }\end{array}$ \\
\hline 5 Matorral en 2003 y Pino en 2006 & 2.06 & 1.38 & $\begin{array}{l}\text { Cuando Matorral perdib, lo reemplaxodel } \\
\text { Pine. }\end{array}$ \\
\hline $\begin{array}{l}\text { Rericultura en } 2003 \text { y Bosque mixto en } \\
\text { aoos }\end{array}$ & -2.87 & -0.83 & $\begin{array}{l}\text { Cuando Agricultura perdib, Bosque mixto } \\
\text { no la reemplazs. }\end{array}$ \\
\hline Agricultura en 2003 y Matorral en 2006 & 3.36 & 1.17 & $\begin{array}{l}\text { Cuanda Agricultura perdio, Matorralla } \\
\text { roemplaza. }\end{array}$ \\
\hline $\begin{array}{l}\text { No Bosque mixtoen } 2003 \text { y Bosque mixto } \\
\text { en } 2006\end{array}$ & -6.10 & -0.60 & $\begin{array}{l}\text { Cuando Categorlas de No Bosque miato } \\
\text { pordieron, Catogorias de No Bosque las } \\
\text { rowmplazaren. Bóque mixto no gano. }\end{array}$ \\
\hline No Matorralen 2009 y Matorral en 2006 & 3.25 & 0.36 & $\begin{array}{l}\text { Cuando Categorlas de No Matorral } \\
\text { perdieron, Matorral los recmplazs. } \\
\text { Matorral fan }\end{array}$ \\
\hline
\end{tabular}

Fuente: Elaboración propia.

De los resultados presentados en la Tabla 8.B interpretamos como las transiciones más sistemáticas en pérdidas:

- Agricultura intervino al Bosque mixto.

- Regeneración del Bosque mixto a partir del Pino.

- Regeneración de Pino a partir de Matorral.

- Regeneración de Matorral a partir de Agricultura. 


\section{DISCUSIONY CONCLUSIONES}

Los resultados del estudio de la detección de cambios realizado en 2012 (Pineda de Carías, Ochoa, \& Corrales, 2012) demandó más profundización, debido a que para algunos investigadores y técnicos era difícil de creer la alta dinámica de cambio presente en el sector estudiado. A nuestro modo de ver, este es precisamente uno de los puntos que Pontius et al (Pontius, Shusas, \& McEachem, 2004) examinan en su trabajo cuando expresaron que, un modelo que predice que no hay cambios es más creíble que uno que predice altos cambios, el cual puede considerarse hasta equivocado. Los modelos se consideran excelentes cuando domina la persistencia, pero se consideran incorrectos cuando predicen cambios. Para ilustrar que la literatura publicada muestra que este fenómeno es más bien la regla que la excepción, ellos refieren a varios autores tales como: Wear and Bolstad (1998), Mertens y Lambin (2000), Goeghegan et al (2001), Schneider y Pontius (2001), Brown et al (2002), Chen et al (2002), Lo y Yang (2002), y Manson (2002).

Como ya se mencionó, utilizando el Método de Pontius et al, en este nuevo trabajo nuestros resultados evidencian una alta dinámica de cambio del paisaje estudiado: $52.57 \%$ entre $1991-1995$ y $48.78 \%$ entre $2003-2006$.

Concluimos señalando que, las principales transiciones sistemáticas que se dieron entre 1991-1995 fueron:

- De Bosque mixto a Pino, que interpretamos fueron debidas a la expulsión de grandes aserraderos de la zona;

- De Matorral a Agricultura, debidas al avance de la frontera agrícola de la zona.

Entre 2003-2006 las principales transiciones se dieron:

- De Pino a Bosque mixto, que interpretamos fueron debidas a la importancia económica que adquirieron otras especies, entre los pobladores de la zona;

- De Matorral a Pino, debidas al agotamiento de la capacidad productiva de matorrales que pasaron a especies de mayor valor económico, como el pino.

Estas últimas tendencias revelan la potencialidad de regeneración natural del bosque e intencionalidades humanas de protección y económicas. 


\section{BIBLIOGRAFÍA}

- Brown, D., Goovaerts, P., Burnicki, A., \& Li, M. Y. (2002). Stochastic simulation of land cover change using geostatistics and generalized additive models. Photogramm. Eng. Remote Sensing, 68(10), 1051-1061.

- Chen, J., Gong, P., He, C., Luo, W., Tamura, M., \& Shi, P. (2002). Assessment of the urban development plan of Beijing by using a CA-based urgan growth model. Photogramm. Eng. Remote Sensing, 68(10), 1063-1071.

- Geoghegan, J., Villar, S. C., Klepeis, P., Mendoza, P. M., Ogneva-Himmelberger, Y., Roy Chowddhury, R., . . Vance, C. (2001). Modeling tropical deforestation in the souther Yucatan peninsular region: comparing survey and satellite data. Ecosyst. Environ., 85((1-3)), 25-46.

- Lo, C. P., \& Yang, X. (2002). Drivers of land-use/land-cover changes and dynamic modeling for the Atlanta, Georgia metropolitan area. Photogramm. Eng. Remote Sensing, 68(10), 1073-1082.

- Manson, S. M. (2002). Integrated assessment and projection of land-use and land-cover change in the Souther Yucatan Peninsular Region of Mexico. Worcester, Massachusetts: Clark University.

- Mertens, B., \& Lambin, E. (2000). Land cover-change trajectories in southern Cameroon. Ann. Assoc. Am. Geogr., 90(3), 467-494.

- Pineda de Carías, M. C., Ochoa, V. L., \& Corrales, R. E. (Diciembre de 2012). Detección de cambios en la cobertura de la tierra de un sector del occidente de Honduras Período 1991-2006. Ciencia y Tecnología(Número 11), 78-109.

- Pineda Portillo, N. (2008). Geografía de Honduras. Tegucigalpa, Honduras: Multigráficos Flores S. de R. L.

- Pontius, R. G., Shusas, E., \& McEachem, M. (2004). Detecting important categorical land changes while accounting for persistance. Agriculture, Ecosystems \& Environment, 101, 251-268. 
- Schneider, L., \& Pontius, R. (2001). Modeling land-ue change in the Ipswich watershed, Massachusetts, USA. Agric. Ecosyst. Environ., 85((1-3)), 83-94.

- Wear, D., \& Bolstad, P. (1998). Land-use changes in southern Appalachian landscapes: spatial analysis and forecast evaluation. Ecosystems , 1, 575-594. 